\title{
Perfil socioepidemiológico e autonomia de longevos em Recife-PE, Nordeste do Brasil
}

\author{
Socio-epidemiological profile and autonomy of elderly in the city of Recife, northeastern Brazil
}

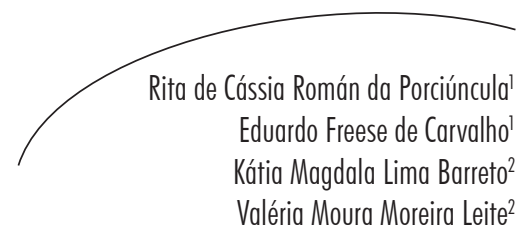

Resumo

Dos processos articulados de transição demográfica e epidemiológica em curso nas últimas décadas no país, decorre o crescimento da população idosa, particularmente do número de idosos longevos, com idade acima de 80 anos. Este processo de envelhecimento da população impõe uma reorganização do Estado e da sociedade, principalmente em relação aos sistemas de saúde e previdenciário. Objetivo: Analisar o perfil socioepidemiológico e o grau de autonomia e independência de idosos longevos na cidade de Recife-PE. Método: Trata-se de um estudo epidemiológico descritivo, do tipo corte transversal, de amostra aleatória, composto por 227 idosos. Estes responderam ao questionário Brazil Old Age Schedule (BOAS) modificado em entrevistas domiciliares nos seis distritos da cidade. Resultados: Na maioria dos idosos, verificaram-se a feminização, o baixo nível de escolaridade, a viuvez como estado conjugal e a aposentadoria como principal fonte de renda. Os idosos residiam comumente com filhas e netos, e os principais problemas de saúde foram hipertensão, doença de coluna, problemas de visão, incontinência urinária e osteoporose. O grau de autonomia e independência dos idosos longevos foi considerado bom, uma vez que não houve relato de impedimentos em realizar as atividades da vida diária (AVD) pela maioria dos idosos. Conclusões: Com a tendência de ampliação do número de idosos longevos nos próximos anos, é necessário implementar políticas públicas específicas, adequando programas e ações que contribuam efetivamente para a obtenção da qualidade de vida na velhice avançada.

\section{Abstract}

Articulated processes of demographic and epidemiological transition in progress in recent decades in the country are followed by the growth of the elderly population, particularly the number of oldest old, aged over 80 years. This population aging requires reorganization of the state and society, especially in relation to health and

\footnotetext{
Programa de pós-graduação em Saúde Pública, Departamento de Saúde Coletiva, Centro de Pesquisa Aggeu Magalhães. Fundação Oswaldo Cruz. Recife, PE, Brasil.

2 Departamento de Terapia Ocupacional, Centro de Ciências da Saúde. Universidade Federal de Pernambuco. Recife, PE, Brasil.
}

Palavras-chave: Idoso de 80 anos ou mais. Longevidade. Envelhecimento da população. Perfil de saúde. Estudos transversais. 
pension systems. Objective: To analyze the socio-epidemiological profile and degree of autonomy and independence of the oldest old in the city of Recife, state of Pernambuco, Brazil. Method: This was a descriptive epidemiological study, of cross-sectional type, with random sample composed of 227 seniors. They answered the questionnaire Brazil Old Age Schedule (BOAS) Modified on household interviews in the six districts of the city. Results: In most elderly there are feminization, low level of education, marital status as widowhood and retirement as main source of income. Older people commonly lived with daughters and grandchildren, and major health problems were hypertension, column disease, vision problems, incontinence and osteoporosis. The degree of autonomy and independence of the oldest old was considered good, since there were no reports of impairments in performing activities of daily living (ADLs) for most seniors. Conclusions: With the trend towards increasing the number of oldest old in the coming years, it is necessary to implement specific policies, adapting programs and actions that contribute effectively to the achievement of quality of life in advanced old age.
Key words: Aged 80 and over. Longevity. Demographic ageing. Health profile. Crosssectional studies.

\section{INTRODUÇÃO}

O processo de envelhecimento populacional baseia-se em grandes transformações políticas e socioeconômicas que geraram mudanças nos perfis demográfico e epidemiológico, ocorridas nas diferentes sociedades, principalmente a partir do século passado. ${ }^{1}$ No Brasil, esse processo ocorre de forma acelerada, associando novas doenças decorrentes do prolongamento da vida às antigas doenças negligenciadas de um contexto de iniquidade social. ${ }^{1}$ Neste panorama, é possível observar alta proporção de sua população na faixa etária jovem e outra parcela significativa da população envelhecida. Os dois grupos têm necessidades médicas e sociais específicas, a serem atendidas com recursos escassos. ${ }^{2}$

Entre as características do envelhecimento da população brasileira está o aumento da proporção de mulheres à medida que os idosos envelhecem. Em 2000, para um grupo de 100 mulheres havia cerca de 81 homens; em 2050, haverá aproximadamente, $76 .^{3}$ Esse fenômeno se acentua entre idosos com mais de 80 anos, chegando a 61 homens para cada 100 mulheres e alcançando a proporção de quase duas mulheres para cada homem. ${ }^{4,5}$

De acordo com estudos realizados, $22 \%$ dos idosos não apresentam doenças crônicas; 23\% apresentam duas doenças e 30\% pelo menos três, resultando que mais da metade dos 30.000 idosos estudados em todo o país possui um quadro de doenças múltiplas. ${ }^{6} \mathrm{~A}$ hipertensão arterial e a artrite são as mais frequentemente relatadas pelas pessoas idosas, mas também destacam-se doenças do coração, diabetes, asma/bronquite, câncer e cirrose. ${ }^{6}$ Vale salientar que diabetes e depressão, apesar de terem prevalências menores, são potencialmente incapacitantes e aumentam os riscos de mortalidade.

Uma das características que torna o perfil epidemiológico dos idosos particular é a predominância de doenças crônicas, de progressão gradual e alta capacidade de afetar e reduzir a autonomia e independência do indivíduo. Em algum momento do adoecimento, o idoso buscará o serviço de saúde e, em geral, o uso é mais prolongado do que em outras etapas da vida. ${ }^{8}$

Dados encontrados em estudos sobre perfil do idoso longevo em Ribeirão Preto-SP e Caxias do Sul-RS, por meio da Avaliação Geriátrica Global, revelam média de idade em torno de 85 anos, predomínio do sexo feminino, da viuvez e de baixos índices de escolaridade. Os homens idosos casados com maior escolaridade e melhor renda apresentam níveis melhores de independência e autonomia. ${ }^{9}$ 
Esse contexto formula, para especialistas, gestores e para o conjunto da sociedade, um dos maiores desafios sociais e intensa demanda por estudos e análises para a melhor definição de políticas públicas de prevenção e promoção da saúde no envelhecimento. ${ }^{10}$ No entanto, grande parte dos estudos aborda exclusivamente as pessoas que têm entre 60 e 80 anos, conformando uma amostra que não leva em conta os idosos longevos, com 80 anos ou mais, parcela da população que tem crescido significativamente. ${ }^{3}$

A implementação de políticas e programas que priorizem o envelhecimento ativo pode mudar o quadro de instalação de doenças crônicas e suas incapacidades, mesmo nas fases mais tardias da vida, além de valorizar a autonomia da pessoa idosa e buscar a manutenção de sua capacidade funcional, garantindo sua participação ativa na sociedade por mais tempo. ${ }^{11,12}$ Para tanto, políticas adequadas precisam ser baseadas em informações sobre a população à qual se destina e ainda são escassas as pesquisas voltadas à população de idosos longevos.

Nesse sentido, este artigo objetivou analisar o perfil socioepidemiológico e o grau de autonomia e independência de idosos com 80 anos de idade ou mais do município de Recife-PE, situado no Nordeste brasileiro.

\section{MÉTODOS}

Trata-se de um estudo epidemiológico descritivo de base populacional e de corte transversal. Para a elaboração deste artigo, foram utilizados dados do estudo "Mobilidade urbana, acesso e saúde: um estudo da população idosa", com 1.200 entrevistados em todo o município. Os dados foram coletados por meio de inquérito domiciliar realizado entre novembro de 2010 e agosto de 2011.

A amostra foi estratificada inicialmente com partilha proporcional, considerando a proporção de pessoas com idade igual ou superior a 65 anos de cada um dos seis distritos sanitários do Recife-PE, chegando-se a um total de 1.200 sujeitos. Além disso, adotou-se também a partilha proporcional em função das faixas etárias: 65 a 69 anos; 70 a 74 anos; 75 a 79 anos; 80 anos ou mais, para cada um dos seis distritos sanitários de Recife, ou seja, cada distrito continha uma proporção específica de idosos correspondente a cada faixa etária. Uma vez definido o número de sujeitos em cada distrito, realizou-se um sorteio aleatório dos setores censitários (SC) nos quais foram realizadas as entrevistas. Por SC, obedeceuse ao número limite de dez entrevistados, sendo visitados quantos setores fossem necessários para contemplar os critérios estabelecidos: o número de idosos por distrito e o número de idosos por faixa etária.

Considerando a amostra, foram entrevistados 227 idosos com 80 anos de idade ou mais. Como critério de exclusão, considerou-se a situação do idoso que morava sozinho com comprometimento cognitivo grave e que não possuísse um responsável e/ou cuidador que pudesse responder ao questionário.

Osparticipantes dapesquisa responderam a um questionário, baseado no modelo Brazil Old Age Schedule (BOAS) modificado, um instrumento multidimensional desenvolvido para pesquisas com populações idosas. ${ }^{13}$ Os dados utilizados se referem às seções de informações gerais (I), saúde física (II), atividades da vida diária (III) e recursos econômicos (IV).

A seção I investigou sexo, nível de escolaridade, estado conjugal, número de filhos e composição geral domiciliar, permitindo conhecer a situação pessoal e familiar do idoso. Para conhecer a saúde e a morbidade referidas, a seção II busca saber como o idoso define sua condição atual de saúde, as doenças relatadas e como percebe a capacidade destas enfermidades de limitar suas atividades rotineiras. A seção III trata da autonomia e independência do indivíduo para a realização de Atividades da Vida Diária (AVD), que contemplam diversas atividades da rotina, tendo os idosos sido questionados a respeito de 
sua capacidade de realizá-las sozinhos. Com o objetivo de conhecer a situação socioeconômica, a seção IV compreende questões sobre a situação de trabalho, a renda mensal do idoso e fonte desta renda, a renda mensal familiar, a situação do imóvel em que vive e a suficiência da renda pessoal para as despesas básicas.

A pesquisa se desenvolveu no domicílio, havendo três possibilidades para o respondente do questionário. O próprio idoso poderia responder diretamente e sem ajuda de terceiros, com ajuda, e o questionário poderia ser respondido totalmente por outra pessoa do domicílio (respondente substituto), sem a participação do idoso, impossibilitado de fazê-lo sozinho e com fidedignidade.

Os dados coletados foram submetidos ao tratamento estatístico por meio de análise bivariada, para verificar a existência de associação (teste qui-quadrado e teste Exato de Fisher). Todos os testes foram aplicados com 95\% de confiança.
Este estudo foi desenvolvido em conformidade com as recomendações éticas, tendo os dados sido cedidos mediante carta de anuência da autora, obtendo aprovação do Comitê de Ética em Pesquisa envolvendo seres humanos do Centro de Ciências da Saúde da Universidade Federal de Pernambuco, sob o protocolo no 315/2010 CEP/CCS. Os idosos que aceitaram participar da pesquisa assinaram o Termo de Consentimento Livre e Esclarecido (TCLE).

\section{RESULTADOS}

Para as possibilidades de resposta, têm-se $83,6 \%$ dos questionários respondidos pelos próprios idosos sem ajuda; $11,0 \%$ por idosos com ajuda e $4,3 \%$ por respondente substituto.

Ao analisar a relação entre sexo e faixa etária, verificou-se predominância do sexo feminino em todas as faixas, principalmente entre 85 e 89 anos (tabela 1). 
Tabela 1. Distribuição dos idosos segundo a faixa etária, estado conjugal, arranjo familiar, escolaridade e saúde referida por sexo. Recife-PE, 2010-2011.

\begin{tabular}{|c|c|c|c|c|c|}
\hline \multirow[t]{2}{*}{ Variável } & \multicolumn{2}{|c|}{ Masculino } & \multicolumn{2}{|c|}{ Feminino } & \multirow[t]{2}{*}{ p-Valor } \\
\hline & $\mathrm{n}$ & $\%$ & $\mathrm{n}$ & $\%$ & \\
\hline Sexo & 52 & 22,6 & 178 & 77,4 & \\
\hline Faixa etária & & & & & $<0,001$ \\
\hline $80-84$ & 30 & 22,39 & 104 & 77,61 & \\
\hline $85-89$ & 09 & 15,79 & 48 & 84,21 & \\
\hline $90-94$ & 10 & 40 & 15 & 60 & \\
\hline $95-99$ & 02 & 18,18 & 09 & 81,82 & \\
\hline 100 ou mais & 01 & 33,33 & 02 & 66,67 & \\
\hline Estado conjugal & & & & & 0,001 \\
\hline Casado/morando junto & 27 & 65,9 & 14 & 34,1 & \\
\hline Viúvos & 19 & 12,8 & 130 & 87,2 & \\
\hline Divorciado & 5 & 41,7 & 7 & 58,3 & \\
\hline Nunca casou/solteiro & 1 & 3,6 & 27 & 96,4 & \\
\hline Arranjo domiciliar & & & & & 0,01 \\
\hline Cônjuge & 27 & 65,9 & 14 & 34,1 & \\
\hline Filho & 15 & 23,8 & 48 & 76,2 & \\
\hline Filha & 21 & 21,6 & 76 & 78,4 & \\
\hline Netos & 19 & 22,6 & 65 & 77,4 & \\
\hline Escolaridade & & & & & 0,37 \\
\hline Nenhuma & 23 & 44,2 & 62 & 35,8 & \\
\hline Primário & 9 & 17,3 & 52 & 30,1 & \\
\hline Ginásio & 6 & 11,5 & 24 & 13,9 & \\
\hline $2^{\circ}$ grau & 8 & 15,4 & 18 & 10,4 & \\
\hline Superior/pós-graduação & 6 & 11,5 & 17 & 9,8 & \\
\hline Saúde referida & & & & & 0,71 \\
\hline Ótima/boa & 32 & 71,1 & 98 & 62,8 & \\
\hline Ruim & 9 & 20 & 43 & 27,6 & \\
\hline Péssima & 4 & 8,9 & 15 & 9,6 & \\
\hline
\end{tabular}


A viuvez é o estado conjugal mais observado entre idosos longevos; no entanto, analisando separadamente o comportamento de homens e mulheres, tem-se que a maioria dos homens era composta por casados $(65,9 \%)$, seguidos de viúvos, enquanto se destacou a viuvez entre as mulheres $(87,2 \%)$, segundo a tabela 1 .

Quanto ao arranjo domiciliar, verificou-se que a composição da maioria dos domicílios variava entre três e cinco pessoas (incluindo o idoso). Identificou-se ainda cerca de $12 \%$ do total de idosos morando sozinhos. Entre as pessoas que residiam com os idosos pesquisados estão, em primeiro lugar, as filhas, seguidas dos netos. A maioria dos homens idosos residia com a esposa $(65,9 \%)$, enquanto $34,1 \%$ das mulheres residiam com os maridos (tabela 1 )

A maioria dos idosos não possuía escolaridade ou concluiu apenas o $1^{\circ}$ grau e uma pequena parcela possuía nível superior ou pós-graduação. Entre os homens, os que não tinham escolaridade representaram uma parcela maior $(44,2 \%)$ e as mulheres estudaram mais do que os homens até o $2^{\circ}$ grau (tabela 1 ).
Em relação à saúde referida, os idosos, em geral, referiram ótimo ou bom nível de saúde. As mulheres fizeram mais referência a ter saúde ruim e péssima $(37,2 \%)$ que os homens $(28,9 \%)$. Quanto à morbidade referida, a hipertensão arterial, a doença de coluna ou dor nas costas e os problemas de visão foram as enfermidades mais referidas. Vale salientar que alcoolismo e cirrose não foram referidos por nenhum entrevistado.

Ao analisar a interferência das doenças na rotina diária dos idosos, 51\% deles afirmaram que os problemas de saúde prejudicavam a realização das atividades que desejavam ou precisavam fazer, mas para $49 \%$ não houve limitação das tarefas por causa de suas enfermidades. Verificou-se que os idosos gozavam de boa autonomia e independência no desempenho de suas tarefas diárias, uma vez que a maioria afirmou desempenhá-las sem ajuda (tabela 2). As atividades nas quais houve maior dificuldade foram sair de casa usando algum tipo de transporte, cortar as unhas dos pés e sair de casa para caminhar curtas distâncias pelo bairro.

Tabela 2. Distribuição dos idosos segundo capacidade de realizarem sozinhos e sem ajuda as Atividades da Vida Diária. Recife-PE, 2010-2011.

\begin{tabular}{lc}
\hline \multicolumn{1}{c}{ Atividade } & $\%$ \\
\hline Comer a sua refeição & 90,4 \\
Pentear os cabelos & 87,8 \\
Deitar e levantar da cama & 86,5 \\
Caminhar em superfície plana & 86,1 \\
Vestir-se & 83,9 \\
Tomar banho & 79,1 \\
Tomas seus remédios & 70,0 \\
Ir ao banheiro a tempo & 67,4 \\
Arrumar sua casa, sua cama & 63,5 \\
Preparar sua própria refeição & 57,0 \\
Subir/descer escada & 56,1 \\
Sair de casa para curtas distâncias & 47,4 \\
Cortar as unhas do pé & 43,0 \\
Sair de casa usando transporte & 39,6 \\
Sair de casa dirigindo seu próprio carro & 13,8 \\
\hline
\end{tabular}


Observou-se que a maioria dos idosos estava aposentada e 28,3\% exerciam alguma atividade no período de realização das entrevistas. Os resultados relativos à fonte de renda mostraram que grande parte devia seu sustento aos rendimentos da aposentadoria e em 43,9\% dos casos a renda se originava de pensão/ajuda do cônjuge. A maioria ganhava um salário mínimo $(\mathrm{SM})$, conforme a tabela 3. Constatou-se que $2 \%$ das idosas relataram receber menos de um SM por mês e que $53,7 \%$ das mulheres idosas percebiam até um SM mensal. Para salários mais altos, os resultados mostram percentuais mais elevados para os homens.

Tabela 3. Distribuição dos idosos segundo renda mensal pessoal, renda mensal familiar, condição de moradia e suficiência da renda própria, por sexo. Recife-PE, 2010-2011.

\begin{tabular}{|c|c|c|c|c|c|}
\hline \multirow[t]{2}{*}{ Variável } & \multicolumn{2}{|c|}{ Masculino } & \multicolumn{2}{|c|}{ Feminino } & \multirow{2}{*}{ p-Valor } \\
\hline & $\mathrm{n}$ & $\%$ & $\mathrm{n}$ & $\%$ & \\
\hline Renda mensal pessoal & & & & & 0,24 \\
\hline Até 1SM & 18 & 37,5 & 80 & 53,7 & \\
\hline$>1 \mathrm{SM}-2 \mathrm{SM}$ & 11 & 22,9 & 19 & 12,8 & \\
\hline$>2 S M-5 S M$ & 11 & 22,9 & 27 & 18,1 & \\
\hline$>5 \mathrm{SM}$ & 8 & 16,7 & 23 & 15,4 & \\
\hline Renda mensal familiar & & & & & 0,09 \\
\hline Até 1SM & 6 & 16,7 & 34 & 28,8 & \\
\hline$>1 S M-2 S M$ & 9 & 25,0 & 25 & 21,2 & \\
\hline$>2 S M-5 S M$ & 14 & 38,9 & 30 & 25,4 & \\
\hline$>5 \mathrm{SM}$ & 7 & 19,4 & 29 & 24,6 & \\
\hline Condição de moradia & & & & & 0,04 \\
\hline Com casa própria & 39 & 75,0 & 103 & 58,2 & \\
\hline Sem casa própria & 13 & 25,0 & 74 & 41,8 & \\
\hline Suficiência da renda para despesas básicas & & & & & 0,29 \\
\hline Suficiente & 32 & 61,5 & 88 & 52,1 & \\
\hline Insuficiente & 20 & 38,5 & 81 & 47,9 & \\
\hline
\end{tabular}

Em relação à renda familiar mensal e o sexo, a maioria dos homens vivia em domicílios com renda média acima de dois SMs (58,3\%), em comparação a $50 \%$ das mulheres na mesma condição. Vale salientar que cerca de $30 \%$ das idosas viviam em domicílios com renda de apenas um SM, ao passo que este percentual na população masculina é de 16,7\%. Quanto à condição de moradia, observa-se que, em geral, homens e mulheres possuíam casa própria e consideraram ter o suficiente para se manter (tabela 3).

\section{DISCUSSÃO}

Nas últimas décadas, o Brasil viveu um crescimento acelerado de sua população idosa, em decorrência dos processos articulados de transição demográfica e epidemiológica. De acordo com a Organização Mundial da Saúde, Recife pode ser considerada uma cidade em processo de envelhecimento, apresentando algumas das características desse fenômeno, como foi possível observar neste estudo. A população 
de idosos já representa cerca de $9 \%$ do total de habitantes, e os idosos com 80 anos de idade ou mais se aproximam de $25 \%$ desta parcela. ${ }^{14}$

Ao analisar os resultados encontrados, verificou-se uma importante feminização da população idosa mais velha, assim como os achados em estudos brasileiros semelhantes. ${ }^{4,15,16}$ A dinâmica das populações idosas masculina e feminina apresenta ritmos diferentes de crescimento, alterados marcadamente por características distintas de mortalidade ligadas ao sexo. Enquanto homens idosos estão mais expostos às doenças letais, como as isquêmicas do coração, as idosas convivem com doenças não fatais, porém crônicas e incapacitantes, como a artrite e a hipertensão. ${ }^{17}$

Por outro lado, características da organização social do trabalho e o comportamento individual em relação ao cuidado com a própria saúde devem ser considerados na medida em que os homens estão mais expostos aos riscos de acidentes de trabalho, ao tabagismo e alcoolismo e à violência, enquanto as mulheres procuram mais os serviços de saúde, fazem mais uso de medicamentos e apresentam maiores taxas de adesão aos programas de prevenção. ${ }^{18}$

Em relação à escolaridade, a maioria dos idosos não estudou ou concluiu apenas o primário. Embora esses resultados não tenham apresentado significância estatísitca, estão de acordo com outros estudos brasileiros que encontram a parcela mais significativa de idosos entre analfabetos e com baixa escolaridade. .,19-22 $^{10}$ Apenas recentemente o Brasil adotou políticas públicas e ações que começaram a modificar o quadro existente. Na população idosa longeva, que viveu o período escolar em que não havia a abrangência do ensino público de massa, esperava-se um nível baixo de escolaridade.

O estado conjugal caracterizado pela predominância de viúvos corrobora os resultados de outros estudos. ${ }^{4,16,21,22} \mathrm{O}$ mesmo acontece na análise da relação entre as variáveis "sexo" e "estado conjugal", verificada como estatisticamente significativa. Enquanto a maioria das mulheres se mantém viúva depois do falecimento do marido, entre os homens predomina o casamento. A viuvez feminina é duas vezes maior que a masculina. Levando-se em consideração o aumento da expectativa de vida do brasileiro, espera-se que mais pessoas cheguem à velhice avançada, porém a mortalidade ainda é maior entre homens em faixa mais jovens, o que faz com que a viuvez seja bastante comum entre as mulheres. ${ }^{20,23,24}$

Os resultados deste estudo mostraram que os idosos acompanham a tendência de morar sozinhos. ${ }^{19,22,25}$ Estudos demonstram o aumento do número de idosos mais velhos morando sozinhos, e entre eles se sobressaem as mulheres. Ainda como influência social neste processo, tem-se a grande mobilidade das famílias, decorrente do aumento de separações dos casais e da construção de novos arranjos, deixando o idoso isolado e muitas vezes sem seu lugar nas novas conformações familiares. ${ }^{18}$

Por outro lado, o arranjo familiar da maioria dos idosos, que não mora só, mostra que eles convivem com as filhas e os netos, achados que não apresentaram significância estatística, mas confirmam dados sobre a composição dos domicílios. ${ }^{19,21}$ Estudos apontam a tendência de que as idosas residam com filhos e netos, enquanto os idosos, com as esposas, ${ }^{26-28}$ possibilitando o aumento da rede de apoio. Apesar de o arranjo familiar se conformar de maneira diferente ao comparar o contexto masculino e feminino, o apoio se fortalece para ambos os sexos com a proximidade dos familiares. Nesse sentido, no Brasil o arranjo multigeracional parece receber mais influência do aspecto econômico do que cultural. Apesar de experimentar o declínio da renda com o avanço da idade, de forma geral os idosos continuam participando significativamente do orçamento doméstico, sobretudo as mulheres. ${ }^{29}$

Quanto à morbidade referida, as doenças crônicas são as mais relatadas, confirmando a hipertensão arterial como morbidade de maior referência entre os idosos brasileiros, seguida de artrite e reumatismo. ${ }^{6,16}$ Vale salientar que essas 
doenças de longa duração têm processo gradual de desenvolvimento e alta capacidade de afetar a autonomia e independência do indivíduo. ${ }^{8}$

O processo de envelhecimento parece criar duas atitudes diferentes frente às limitações e incapacidades. A primeira é uma adaptação necessária às mudanças à medida que elas vão ocorrendo, e estas não se dão de forma rápida e abrupta na maioria das vezes. E a segunda atitude é a de maior aceitação destas mudanças, uma vez que elas são parte de um envelhecer natural, de acordo com uma visão socialmente construída. Isso sugere que os idosos associam a perda da capacidade funcional ao envelhecimento e não à perda da saúde. Estudos demonstram que a capacidade funcional tem sido mais significativa na vida dos indivíduos do que a presença de doenças. A capacidade de mudar a autoavaliação do nível de saúde está mais relacionada à diminuição da capacidade funcional pela presença de uma doença sem controle. ${ }^{30}$

No que se refere às Atividades da Vida Diária (AVD), é possível verificar que os idosos gozam de boa autonomia e independência no desempenho de suas tarefas, uma vez que a maioria afirma desempenhá-las sem ajuda. Estudos realizados no Sudeste do país mostram resultados semelhantes. ${ }^{11,16}$

Em relação aos recursos econômicos, os resultados deste estudo não apresentaram significância estatística, mas demonstraram os baixos rendimentos pessoais dos idosos, principalmente das mulheres. A aposentadoria predomina como a principal fonte de renda. Além dela, existem também as pensões constituindo a renda do idoso.

Nota-se a grande participação do benefício previdenciário para compor o rendimento mensal na velhice avançada, conforme achados de estudos brasileiros. ${ }^{30}$ Apesar de ainda possuir um valor questionável quanto à cobertura das necessidades básicas, os benefícios previdenciários, entre eles o Benefício de Amparo ao Idoso, garantem uma condição mínima de autonomia financeira.
A situação da renda familiar também repercute neste quadro. Os resultados mostram uma condição econômica mais favorável para os homens idosos que viviam em residências com renda média acima de dois SMs, além de usufruírem da propriedade do imóvel habitado em 75\% dos casos, dados estatisticamente significativos. Possuir casa própria significa menos despesas no orçamento doméstico e mais conforto às pessoas na velhice.

Este estudo tem como limitação não ter analisado e comparado o perfil dos idosos longevos em relação aos idosos mais jovens, para quantificar as perdas ocorridas no processo de envelhecimento avançado. Também não foi possível avaliar o impacto das variáveis "escolaridade", "sexo" e "condições de vida na condição de saúde".

\section{CONCLUSÃO}

A metodologia e a análise dos resultados obtidos neste estudo permitiram encontrar respostas que contemplam as questões colocadas inicialmente, confirmando, na população estudada, perfil semelhante ao descrito por estudos que abordam as tendências do envelhecimento populacional brasileiro.

Em relação à caracterização do grupo de idosos longevos e sua situação econômica, há predominância do sexo feminino, principalmente nasidades mais avançadas. O nível de escolaridade é baixo e o estado conjugal mais comum é a viuvez. Em grande parte, seus rendimentos mensais pessoais são de cerca de um salário mínimo, renda originária da aposentadoria do Instituto Nacional de Seguridade Social (INSS).

Os principais problemas de saúde referidos são hipertensão, doença de coluna ou dor nas costas, problemas de visão, incontinência urinária e osteoporose. Metade dos idosos entrevistados acredita que as doenças interferem em sua rotina de atividades, limitando-as.

O grau de autonomia e independência dos idosos longevos foi considerado bom, uma vez 
que na maioria das atividades não há relato de dificuldade de realização.

Por fim, nesta fase da vida, a presença de doenças não remete direta e exclusivamente à perda de autonomia e independência. Logo, abordar a longevidade por meio do enfoque da capacidade funcional e do envelhecimento ativo traz mais benefícios aos idosos e requer uma reavaliação da visão cristalizada sobre o idoso por parte dos profissionais de saúde, da sociedade e do governo.

\section{REFERÊNCIAS}

1. Freese E, Fontbonne A.Transição epidemiológica comparada: modernidade, precariedade e vulnerabilidade. In: Freese E, organizador. Epidemiologia, políticas e determinantes das doenças crônicas não transmissíveis no Brasil. Recife: Ed. Universitária da UFPE; 2006. p.17-45.

2. Veras RP. País jovem com cabelos brancos: a saúde do idoso no Brasil. 2 ed. Rio de Janeiro: UERJ; 1994.

3. Wong RLR, Carvalho JAM, Pertétuo IHO. A estrutura da população brasileira no curto e médio prazos: evidências sobre o panorama demográfico com referência às políticas sociais, particularmente as de saúde. In: Rede Interagencial de Informações para Saúde. Demografia e saúde: contribuição para análise de situação e tendências. Brasília, DF: OPAS; 2009. p.37-63. (Série G. Estatística e Informação em Saúde). (Série Informe de Situação e Tendências).

4. Morais EP de. Envelhecimento no meio rural: condições de vida, saúde e apoio dos idosos mais velhos de Encruzilhada do Sul. [tese] São Paulo: Universidade de São Paulo; 2007.

5. Liposcki DB. A influência de um programa de intervenção psicomotora na aptidão psicomotora de idosos longevos. [dissertação] Santa Catarina: Universidade do Estado de Santa Catarina; 2007.

6. Lima-Costa MF, Barreto SM, Giatti L. Condições de saúde, capacidade funcional, uso de serviços de saúde e gastos com medicamentos da população idosa brasileira: um estudo descritivo baseado na Pesquisa Nacional por Amostra Domiciliar (PNAD 98). Cad Saúde Pública 2003;19(3):735-43.
As informações apresentadas constituem um perfil da população do estudo e generalizações devem ser feitas com muita cautela. No entanto, permitem contribuir com noções específicas e atuais sobre um grupo de idosos longevos. Face ao crescimento progressivo da parcela mais velha da população, é necessário que a sociedade desenvolva o conhecimento detalhado desta etapa da vida, de suas necessidades e particularidades para a adoção de medidas adequadas de cuidado.

7. Lima-Costa MF. O envelhecimento populacional e suas repercussões para a saúde pública. In: Rede Interagencial de Informações para Saúde. Demografia e saúde: contribuição para análise de situação e tendências. Brasília, DF: OPAS; 2009. p.65-82. (Série G. Estatística e Informação em Saúde). (Série Informe de Situação e Tendências).

8. Rede Interagencial de Informações para Saúde. Demografia e saúde: contribuição para análise de situação e tendências. Brasília , DF: OPAS; 2009. Informe de situação e tendências: demografia e saúde. p. 17-34. (Série G. Estatística e Informação em Saúde). (Série Informe de Situação e Tendências).

9. Rosset I. Avaliação geriátrica global dos idosos mais velhos residentes em Ribeirão Preto (SP) e Caixas do Sul (RS): indicadores para envelhecimento longevo [tese]. São Paulo: Universidade de São Paulo; 2009.

10. Motta LB, Aguiar AC. Novas competências profissionais em saúde e o envelhecimento populacional brasileiro: integralidade, interdisciplinaridade e interssetorialidade. Ciênc Saúde Coletiva 2007;12(2):363-72.

11. Parahyba MI, Veras R, Melzer D. Incapacidade funcional entre mulheres idosas no Brasil. Rev Saúde Pública 2005;39(3):383-91.

12. Veras RP, Caldas CP, Coelho FD, Sanchez MA. Promovendo a saúde e prevenindo a dependência: identificando indicadores de fragilidade em idosos dependentes. Rev Bras Geriatr Gerontol 2007;10(3):355-70. 
13. Veras RP, Dutra S. Perfil do idoso brasileiro: questionário BOAS. Rio de Janeiro: UERJ/ UnATI; 2008.

14. Organização Mundial de Saúde. Grupo Científico sobre la Epidemiología del Envejecimiento. Aplicaciones de la epidemiologia al estúdio de los ancianos. Ginebra:OMS;1984. (Série de Informes Técnicos 706)

15. Francisco CM. Estudo dos fatores preditores do envelhecimento sem incapacidade funcional entre idosos em velhice avançada no Município de São Paulo. [dissertação]. São Paulo: Universidade de São Paulo; 2006.

16. Heluany CCV. Perfil do envelhecimento de octogenários e nonagenários residentes em Siderópolis - Santa Catarina. [dissertação] Criciúma: Universidade do Extremo Sul Catarinense; 2007.

17. Berquó, E. Algumas considerações demográficas sobre o envelhecimento no Brasil. Seminário Internacional sobre o Envelhecimento Populacional; 1996; Brasília. Brasília: Ministério da Previdência e Assistência Social; 1996.

18. Veras RP, Ramos LR, Kalache A. Crescimento da população idosa no Brasil: transformações e consequências na sociedade. Rev Saúde Pública 1987;21(3):225-33.

19. Ferreira FFP, Izzo H, Jacob Filho W. Impacto da capacidade física na saúde percebida entre idosos em velhice avançada. Rev Saúde Coletiva 2007;4(17):154-57.

20. Inouye K, Pedrazzani ES. Nível de instrução, status socioeconômico e avaliação de algumas dimensões da qualidade de vida de octogenários. Rev Latino-Am de Enfermagem 2007; 15 n. esp:742-7.
21. Liposcki DB. A influência de um programa de intervenção psicomotora na aptidão psicomotora de idosos longevos. [dissertação] Santa Catarina: Universidade do Estado de Santa Catarina; 2007.

22. Rosset L, Roriz-Cruz M, Santos JLF, Haas VJ, Fabrício-Wehbe SCC, Rodrigues RAP. Diferenciais socioeconômicos e de saúde entre duas comunidades de idosos longevos. Rev Saúde Pública 2011;45(2):391-400.

23. Buaes CS, Doll J. Aprende a ser viúva: narrativa de mulheres idosas no meio rural. Rev Kairós 2005;8(2):171-88.

24. Instituto Brasileiro de Geografia e Estatística. Censo demográfico 2000. Rio de Janeiro: IBGE; 2002.

25. Camarano AA. Envelhecimento da população brasileira: uma contribuição demográfica. Rio de Janeiro: IPEA;2002.

26. Duarte YAO. Desempenho funcional e demandas assistenciais. In: Lebrão ML, Duarte YAO, organizadores. Projeto SABE no município de São Paulo: uma abordagem inicial. Brasília: OPAS; 2003. p.183-200.

27. Ramos LR. Fatores determinantes do envelhecimento saudável em idosos residentes em centro urbano: Projeto Epidoso, São Paulo. Cad Saúde Pública 2003;19(3):793-8.

28. Saad PM. Arranjos familiares e transferências de apoio informal. In: Lebrão ML, Duarte YAO, organizadores. Projeto SABE no município de São Paulo: uma abordagem inicial. Brasília: OPAS; 2003. p.183-200.

29. Sugahara GTL. O perfil do idoso brasileiro. Rev Kairós 2005;8(2):51-75.

30. Alves LCA, Leite IC, Machado CJ. Perfis de saúde dos idosos Brasil: análise da Pesquisa Nacional por Amostra de Domicílios de 2003 utilizando o Método de Grade of Membership. Cad Saúde Pública 2008;24(3):535-46. 